\title{
Improving critical care discharge summaries: a collaborative quality improvement project using PDSA
}

\author{
Lucy Goulding, Hannah Parke, Ritesh Maharaj, Robert Loveridge, Anne McLoone, Sophie Hadfield, Eloise Helme, Philip Hopkins, Jane \\ Sandall \\ King's Improvement Science, King's College London and King's College Hospital NHS Foundation Trust; UK
}

\begin{abstract}
Around 110,000 people spend time in critical care units in England and Wales each year. The transition of care from the intensive care unit to the general ward exposes patients to potential harms from changes in healthcare providers and environment. Nurses working on general wards report anxiety and uncertainty when receiving patients from critical care.
\end{abstract}

An innovative form of enhanced capability critical care outreach called 'iMobile' is being provided at King's College Hospital (KCH). Part of the remit of iMobile is to review patients who have been transferred from critical care to general wards. The iMobile team wished to improve the quality of critical care discharge summaries.

A collaborative evidence-based quality improvement project was therefore undertaken by the iMobile team at $\mathrm{KCH}$ in conjunction with researchers from King's Improvement Science (KIS). Plan, Do, Study, Act (PDSA) methodology was used. Three PDSA cycles were undertaken.

Methods adopted comprised: a scoping literature review to identify relevant guidelines and research evidence to inform all aspects of the quality improvement project; a process mapping exercise; informal focus groups / interviews with staff; patient story-telling work with people who had experienced critical care and subsequent discharge to a general ward; and regular audits of the quality of both medical and nursing critical care discharge summaries.

The following behaviour change interventions were adopted, taking into account evidence of effectiveness from published systematic reviews and considering the local context: regular audit and feedback of the quality of discharge summaries, feedback of patient experience, and championing and education delivered by local opinion leaders.

The audit results were mixed across the trajectory of the project, demonstrating the difficulty of sustaining positive change. This was particularly important as critical care bed occupancy and through-put fluctuates which then impacts on work-load, with new cohorts of staff regularly passing through critical care. In addition to presenting the results of this quality improvement project, we also reflect on the lessons learned and make suggestions for future projects.

\section{Problem}

Critical care areas in acute hospitals are for patients whose condition is life threatening and who require continuous support, treatment, and monitoring. Staffing levels are higher than on general wards and specialist equipment and expertise is available. Patients may be admitted to critical care beds when they are seriously acutely unwell (unplanned or emergency admissions) or following major surgery (planned or elective admissions).

Nationally, there is excess demand for critical care beds. The introduction of the iMobile (critical care outreach and referrals) service at King's College Hospital NHS Foundation Trust $(\mathrm{KCH})$ in September 2013 was designed to alleviate some of the problems relating to high admission rates to critical care and anxieties around detecting and caring for the deteriorating patient. iMobile has four key functions:

1. Rapid response to deteriorating patients on general wards followed by stabilisation and transfer to critical care

2. Proactive outreach advisory and education service for staff on general wards

3. Delivery of mobile critical care interventions and expertise on general wards (rather than within designated critical care areas)

4. Critical care discharge follow-up on general wards.

The iMobile team review every patient that has been discharged from critical care to a general ward within 48 hours of their discharge. The iMobile team wished to improve handover from critical care to a general ward as handover can be a point for loss of information. Every patient leaving critical care is required to have both a medical and a nursing handover which includes a verbal summary and a written discharge summary that passes on important information about the patient, their stay in critical care, and their on-going care requirements to the receiving ward team. 
However, boxes on the written summary may be left blank when the health professional completing the discharge summary is not certain of the information required; full descriptions are not always given, and relevant information may be missing while potentially irrelevant information is included. Discharge summaries are sometimes completely missing. Staff on general wards cannot provide high quality, safe care to patients who have been critically unwell if they do not have the correct information and plan of care.

\section{Background}

Approximately 110,000 people spend time in critical care units in England and Wales each year.[1] More than two-thirds of these people survive to go home. The experience of critical care discharge for both patients and their families can be poor, and life following discharge can be challenging. Numerous studies suggest that patients are often particularly concerned at the point of discharge from a critical care environment to a general hospital ward.[2-5]

Staff on general wards who receive patients from critical care report feeling ill prepared to take over the care of a patient who has been recently critically ill, particularly if limitations are perceived in the handover and information contained within the case notes.[6,7] Communication barriers which result in a poor handover have frequently been named as a significant contributor to errors and adverse events.[8-13] A recent systematic review documents the challenges of safely discharging patients from critical care. A common theme drawn from synthesis of 244 papers was the importance of compiling complete and accurate information at the point of discharge to facilitate continuity of care.[14]

NICE guidelines for critical care stipulate the need for a formal, structured handover at the transition from critical care to a general ward that is individualised according to the patient's needs and is supported by a written plan.[1,15] The NICE guidelines recommend that the plan includes:

- A summary of the critical care stay, including diagnosis and treatment

- A monitoring and investigation plan

- A plan for on-going treatment, including drugs and therapies, nutrition plan, infection status, and any agreed limitations of treatment

- Physical and rehabilitation needs

- Psychological and emotional needs

- Specific communication or language needs.

Thus there is an extensive evidence base to demonstrate the importance of high quality critical care discharge summaries and handover in delivering high quality, safe care. Therefore, a collaborative evidence-based quality improvement project was set up by iMobile staff at $\mathrm{KCH}$ in conjunction with researchers from
King's Improvement Science (KIS) with the aim of improving critical care discharge summaries locally and sustaining this improvement.

What are we trying to accomplish?

The KIS iMobile project group worked together over a ten month period to improve the availability, completeness and quality of the nursing and medical discharge summaries of patients who are discharged from critical care areas. The project aim was for all discharge summaries to be available and rated as good or excellent by September 2014. Trust authorisation was provided for the project and managerial support was given.

\section{Baseline measurement}

Prior to the collaboration with King's Improvement Science, critical care staff at $\mathrm{KCH}$ had begun the process of improving critical care discharge summaries, but had not measured whether any difference was made as a result of the changes. Work had been undertaken to improve the written communication of treatment limitations, particularly those instituted during the spell in critical care, and to improve medicines reconciliation at the point of discharge. The KIS iMobile project team therefore devised a simple five point rating scale (with the categories: poor; somewhere between poor and good; good; somewhere between good and excellent; and excellent), to audit the availability and perceived quality, accuracy and completeness of both nursing and medical discharge summaries (please see supplementary material). This rating scale was adapted from the Electronic Discharge Summary Systems Self-Evaluation Toolkit [16] following a scoping literature review. The audit tool was used to assess the nursing and medical critical care discharge summaries of every patient that had been discharged from critical care to a general ward within a defined two week audit period.

The audits were undertaken by experienced senior nurses from the iMobile (critical care outreach) team in real-time whilst they conducted their reviews of discharged patients. The contemporaneous timing of the audit with the discharge review is important to note, since instances where the discharge summary is recorded as missing or rated poorly reflect a situation in which a patient is being cared for by a ward team who do not have the correct information and plan of care.

The baseline audit of nursing and medical critical care discharge summaries was undertaken in the last two weeks of May 2014. Discharge summaries relating to 38 patients were audited. Please see supplementary material for results tables.

Baseline audit of nursing critical care discharge summaries:

The baseline audit demonstrated that three out of $38(8 \%)$ nursing critical care discharge summaries were missing at the time of the patient's discharge review. A composite score of quality, accuracy and completeness demonstrated that $70 \%$ of nursing discharge summaries were achieving the required target of 'good' or 'excellent'. Therefore $22 \%$ of nursing discharge summaries that were available and audited did not meet the required standard, and 
of these $4 \%$ were rated as poor.

Baseline audit of medical critical care discharge summaries:

The baseline audit showed that zero out of $38(0 \%)$ medical critical care discharge summaries were missing at the time of the patient's discharge review. However, medical discharge summaries should be available on both the Electronic Patient Record (EPR) and within the notes: four medical discharge summaries were present on EPR but absent from the notes and four medical discharge summaries were present in the notes but absent on EPR. A composite score of quality, accuracy and completeness demonstrated that $97 \%$ of medical discharge summaries were meeting the target standard of 'good' or 'excellent'. A rating of 'somewhere between poor and good' was given to $3 \%$ of summaries. No medical discharge summaries were rated as poor.

Baseline audit of time taken to gather information from discharge summaries prior to reviewing the patient:

The baseline audit showed that the mean time taken by the iMobile nurses to find and read the critical care nursing and medical discharge summaries / patient notes in order to gather the information needed to review the patient was 11.2 minutes (median time 10 minutes).

See supplementary file: ds4886.pdf - "KIS iMobile - Critical care discharge summary audit tool"

\section{Design}

We selected behaviour change interventions to encourage good discharge summary completion after a review of the literature (in particular taking into account evidence of effectiveness from systematic reviews using the 'Rx for Change' database) [17] and discussions amongst the clinical team about what would work best in the local context and be sustainable. The following interventions were therefore selected and delivered to medical and nursing staff working in critical care.

- Regular audit and feedback of the availability and quality of discharge summaries

Two [18,19] out of four [18-21] high quality literature reviews conclude that audit and feedback is an effective behaviour change tool. Audit and feedback is thought to be most effective when: delivered promptly, combined with educational meetings, delivered by a respected person, and offered with a financial incentive.[17]

How was this implemented?

The results of the discharge summary audits were synthesised and disseminated to staff by email at the end of each PDSA cycle. Results of the audits were also presented by local opinion leaders during educational sessions. A financial incentive (£5 GBP shopping voucher) was awarded during PDSA cycles for the staff member who completed the best discharge summary.
- Feedback of patient experience. We are not aware of any high quality systematic review of the effect of feedback of patient experience on health professional behaviour change. However, the project team thought that this would be useful motivator as it was thought that some of the critical care staff lacked awareness of the patient experience at discharge.

How was this implemented?

Five patient accounts were collected by a researcher from King's Improvement Science when patients who had experienced critical care and subsequent transfer to a general ward attended their critical care follow up appointment. The patients were opportunistically sampled. The anonymised accounts were fed back to critical care staff with patients' written consent.

- Championing by local opinion leaders

Local opinion leaders are people who are identified by their colleagues as being influential. A high quality systematic review concludes that local opinion leaders are effective in creating behaviour change, either alone or in combination with other interventions.[22] However, a second high quality systematic review did not include enough studies to be able to draw conclusions.[23]

How was this implemented?

A critical care Matron and a number of critical care doctors (who were part of the KIS iMobile project team) offered staff regular informal support and education on the importance and content of good critical care discharge summaries.

\section{- Delivery of educational sessions}

Two high quality systematic reviews have shown that educational outreach (where a trained professional delivers teaching to individuals in their clinical setting) is effective in creating behaviour change.[24, 25] One review had mixed results.[26] One high quality review concludes that educational meetings (held with groups of people) are effective in creating behaviour change.[27] However, four high quality reviews demonstrate mixed results and a further ten reviews gathered insufficient evidence to draw conclusions.[17]. Educational sessions seem to be more effective if they are interactive.[17]

How was this implemented?

Both educational outreach (with individuals) and educational meetings (with groups) were undertaken. They were led by experienced senior critical care staff. Results of the audits were presented and examples of good quality discharge summaries were discussed. The sessions covered the requirements of good discharge summaries and the importance of this.

\section{- Multifaceted}

Use of two or more interventional strategies is generally considered to be more effective than stand-alone interventions,[28] thus the 
project group adopted audit and feedback, feedback of patient accounts, championing by local opinion leaders and education. These interventions were introduced across the course of three PDSA cycles.

\section{Strategy}

The Model for Improvement (which incorporates Plan, Do, Study, Act (PDSA) methodology) was used to introduce regular audits and behaviour change interventions in a systematic fashion.[29] A Gantt chart was constructed at the outset of the project to facilitate project management. Three PDSA cycles were undertaken over the course of a ten month project.

PDSA cycle 1: The planning phase comprised: a scoping literature review to identify relevant guidelines and research evidence to inform all aspects of the quality improvement project; the development of a logic model to theorise about the links between processes (what critical care staff were doing) and outcomes (eg length of stay, mortality, patient experience) and to identify gaps in the service; and a process mapping exercise to visually represent the transfer of a patient from critical care to a general ward and capture the intricacies of the barriers and enablers to effective handover of a written plan.

The first audit of medical and nursing critical care discharge summaries was undertaken in May 2014. The results of this audit were fed back to all staff working in critical care by email report and through educational sessions delivered at audit days and via championing by local opinion leaders.

PDSA cycle 2: Problems surrounding the medical and nursing critical care discharge summaries were further explored via conversations with a broad range of staff and supported by literature reviews. The literature review suggested that the existing templates for both the medical and nursing critical care discharge summaries covered the important areas as recommended by NICE. However, the feasibility of creating an electronic version of the nursing critical care discharge summary was examined. The nursing and medical discharge summaries of patients leaving critical care at $\mathrm{KCH}$ are separate. The medical discharge summary is electronic and part of the electronic patient record, whereas the nursing discharge summary is paper based and attached to the notes. This can make it difficult for staff on general wards to synthesise important information about patients who have recently been critically unwell. Some staff members proposed that the nursing discharge summary could be electronic in line with the medical discharge summary. The reason for the paper based system was that historically there had been lack of computers in critical care. More computers had been introduced, making an electronic nursing discharge summary feasible. The practicalities of an integrated doctor-nurse discharge summary were also discussed and the evidence base for this was reviewed. However, it was decided that having one integrated form would be logistically awkward. It would be unclear as to who had final responsibility for uploading the form, and there may be ambiguities as to whether the form had been properly signed-off by both the doctor and nurse concerned.
An informal focus group was undertaken with 15 critical care nurses in order to ascertain their views on the quality of discharge summaries being produced, as well as the barriers to good completion. Many of the critical care nurses did not feel that the introduction of an electronic discharge summary was a practical solution at that point in time due to the fact that all of the critical care units at $\mathrm{KCH}$ were due to move over to a bespoke electronic clinical information system in a matter of months.

Given the importance of these local contextual factors it was decided that the nursing critical care discharge summary would not be made electronic at this stage, but that this change could be adopted in a later PDSA cycle if suitable.

During the second PDSA cycle, story-telling work with two patients who had experienced transfer from critical care to general wards was conducted. Patients were invited to talk to a researcher from King's Improvement Science about their experience of transfer from critical care to a general ward when attending their follow up appointment. In line with published literature, the story-telling work revealed that transfer from critical care to a general ward caused anxiety and feelings of uncertainty surrounding the ward team's ability to deliver their care. The anonymised patient stories were fed back to staff working in critical care at $\mathrm{KCH}$ by email with the patients' written consent. Anecdotally, staff reported that patient stories were an important motivator.

The second two week audit of nursing and medical critical care discharge summaries was undertaken in July 2014 and the results were fed back to all staff via an email report and through presentations at audit meetings and team days. Educational sessions on the importance and content of good discharge summaries were concurrently presented at the audit day and in team meetings to support this feedback.

PDSA cycle 3: Further story-telling work with three patients was conducted by a researcher from King's Improvement Science during the third PDSA cycle and these anonymised accounts were fed back to critical care staff with the patients' written consent.

The third PDSA cycle started in the last week of July 2014, thus this cycle included the rotation of new junior doctors into critical care in the first week of August 2014. Specific educational sessions on the importance and content of good critical care discharge summaries were conducted during the induction of the new junior doctors and additional support was provided by local opinion leaders in person and via email reminders as it was widely anticipated that the quality of discharge summaries would decline unless targeted support was given.

The third two week audit of nursing and medical critical care discharge summaries was undertaken in September 2014. The results of the audit were fed back to all critical care staff via email report and continued to be publicised and championed through educational sessions delivered by local opinion leaders at audit days and during team meetings.

The project team are now in the process of planning for further and 
sustained improvement. Important areas to be explored in future PDSA cycles or projects include reducing the number of critical care discharges that take place between 22:00 and 07:00 hours, reducing delayed discharge, preventing re-admission, reintroducing patient-held discharge summaries, and sending critical care discharge summaries to primary care providers.

\section{Results}

In total, three audits of nursing and medical critical care discharge summaries were undertaken by experienced nurses working for the iMobile (critical care outreach) team in line with three PDSA cycles. Each audit form captured information about the quality of one patient's nursing and medical discharge summary, with 101 patients' discharge summaries audited across the three PDSA cycles.

Baseline audit period: 19/05/14 - 01/06/14 (38 audit forms returned)

Second audit period: 10/07/14 - 24/07/14 (34 audit forms returned)

Third audit period: 01/09/14 - 14/09/14 (29 audit forms returned)

The project aim was for $100 \%$ of nursing and medical critical care discharge summaries to be available and to meet the target of being rated as good or excellent quality by September 2014. This target was not met and results across the trajectory of the three audits are mixed demonstrating the difficulty of introducing and sustaining positive change. Please see supplementary material for results tables.

The nursing critical care discharge summaries showed an initial improvement between audits one ( $70 \%$ meeting target) and two (82\% meeting target), followed by a decline at audit three $(70 \%$ meeting target). The decline at audit three was compounded by the high proportion of missing nursing discharge summaries $(17 \%$ missing). Nursing discharge summaries were also missing at audits one and two ( $8 \%$ and $3 \%$ missing respectively). A consistent problem with nursing critical care discharge summaries was the absence of signatures from nurses on the sending and in particular the receiving wards. Signatures are important in demonstrating that a good handover has been delivered or received and understood.

The medical discharge summaries were initially of a high standard with $97 \%$ meeting the target of good or excellent. This had declined by the second audit ( $83 \%$ meeting target), with some improvement shown at the third audit ( $88.5 \%$ meeting target) but without returning to the high level observed at baseline. Across the three audits, no medical discharge summaries were rated as 'poor', but a small proportion were rated as 'somewhere between poor and good'. The decline in the quality of medical discharge summaries during audit two was affected by missing discharge summaries $(6 \%$ missing). No medical discharge summaries were missing at audits one and three.

The iMobile nurses were asked to record the time taken to find and read the nursing and medical discharge summaries in order to gather the information needed to review the patient for each critical care discharge review undertaken during the audit period. The hypothesis was that if the quality of discharge summaries improved, the iMobile team would spend less time searching for information and would then have more time available to spend with the patient. The data for time taken to review discharge summaries is positively skewed with most reviews taking a relatively short time and a small number of reviews taking a longer time. It is therefore necessary to look at the median time to review across the three audits: this did not change and remained consistent at 10 minutes.

The iMobile nurses were asked to provide written free text comments on the quality of each nursing and medical discharge summary reviewed. While these qualitative comments cannot be assessed as indicators of change, they do provide important context and can be used to inform future PDSA cycles. For example, a common comment was that nursing discharge summaries were often filed in the wrong place making them difficult to find. Future educational sessions should include instruction on where to file the summary. The free text comments also reveal ongoing concerns that a small number of nursing discharge summaries are illegible or have sections that are not completed.

The results suggest that nursing critical discharge summaries in particular require further overall improvement. A focus on ensuring that discharge summaries are firstly written and secondly handed over and filed correctly is recommended to enable the ward team to have access to important information about patients' stay in critical care.

The critical care units at $\mathrm{KCH}$ are due to move to a new bespoke electronic clinical information system in the next several months. This quality improvement project will be continued by staff at $\mathrm{KCH}$ with the aim of achieving good quality discharge summaries before, during, and after transition to the new electronic system.

See supplementary file: ds4905.pdf - "KIS iMobile - Results tables"

\section{Lessons and limitations}

Despite being unable to demonstrate continuous and sustained improvement across the three audits that have been undertaken to date, we are able to consider the impact of the local context on the results, critically reflect on the methods adopted, and make recommendations for future PDSA cycles and related quality improvement projects.

Levels of bed occupancy and throughput are likely to affect the workload of staff in critical care and therefore the quality of discharge summaries. Local data demonstrated a huge increase in demand for critical care beds during 2014, particularly over the winter months. Future cycles and projects should attempt to explore the impact of this. Furthermore, staff have suggested that the mixed results observed may be due to regular changes in a significant proportion of the critical care staff due to job rotation, the use of agency and bank staff, or high turnover caused by the pressures of the environment. This highlights the challenge in developing organisational memory and the need to work towards continuous improvement rather than conducting a project that has a defined 
end point.

However, we do not have enough data points at present to be able to conclude with confidence that either bed occupancy / throughput or staff rotations are linked to fluctuations in the quality of discharge summaries as this pattern may be due to some other confounding factor. Thus a key lesson learned from this project is the need for regular (and preferably continuous) measurement throughout PDSA cycles in order to better understand the trends observed. Our suggestion would be to create a shortened version of the audit that asks the auditor to record whether the medical and nursing discharge summaries were good, poor or missing. This information could be collected for every discharge review carried out by the iMobile team and recorded on the Electronic Patient Record, allowing for continuous monitoring of the quality of medical and nursing critical care discharge summaries (rather than at five week intervals).

The construction of run-charts would allow the team to better monitor and understand trends, for example examining whether the quality of discharge summaries dips when a new cohort of staff begin working in critical care and how long it then takes for the discharge summaries to meet the required standard. Educational sessions and mentoring could then be focussed during this period. Continuous measurement would therefore create a sustainable and informative quality improvement project.

The three audits of medical and nursing discharge summaries were undertaken by experienced senior nurses from the iMobile (critical care outreach) team. It appears that medical critical care discharge summaries are of a consistently higher standard than nursing critical care discharge summaries. However, it is possible that the medical discharge summaries were rated consistently higher than the nursing discharge summaries because of the professional experience of the nurses who were undertaking the audit (eg they may have been more aware of what to look for and therefore more critical of their own profession). Alternatively, the apparent difference in the quality of the medical and nursing summaries may be due to the fact that the medical summary is electronic making it easier to find and read than the paper based nursing summary. Furthermore, we were aware at the outset that asking the iMobile nurses to rate the quality of discharge summaries as part of a quality improvement project may have created a Hawthorne effect whereby ratings improve because this is what we hope will happen. However, the results do not show evidence of a Hawthorne effect as there was not a consistent trend towards improvement.

The medical critical care discharge summaries were arguably of a high standard at the start of the project, which left relatively little scope for improvement. However, anecdotal evidence had suggested that the quality of medical discharge summaries could be variable and dependent upon the cohort of junior medical staff currently working within the critical care units. Again, continuous measurement would help to establish whether the quality of discharge summaries is following another local trend.

This project could have been strengthened by introducing process measures to better understand the utility of the educational / feedback sessions that were delivered. For example, measurement of knowledge, attitudes, and beliefs about the content and purpose of critical care discharge summaries could be conducted before and after educational sessions. This would help to infer whether the implementation of educational sessions is better or worse at any given stage of the project, and whether the educational sessions lead to change in practice.

If practicable, we also recommend collecting data for clinical outcome measures, for example length of hospital stay, readmission to critical care and mortality, and other important outcomes such as patient and staff experience. Even so, it should be remembered that it is difficult to establish a causal pathway without a control group (for example, determining whether improved discharge summaries lead to better patient outcomes). In this instance we rely on the evidence base which demonstrates the importance of good critical care discharge summaries in providing high quality, safe care.

We suggest that future project teams explore the feasibility of applying a behaviour change theory or framework. During PDSA cycle 2 we explored the possibility of changing the nursing discharge summary from a paper to an electronic version. At this point in time there was strong resistance to this from nurses working in critical care; in part because of the impending clinical information system due to launch in a number of months' time and in part due to a general reluctance to do things differently. Behaviour change theory and techniques may have helped us to better understand this resistance and support positive change. This issue also points towards the importance of carefully considering the local context and barriers to change in any quality improvement project. Our process mapping exercise undertaken during PDSA cycle 1 was helpful in this regard.

Work had been done at $\mathrm{KCH}$ to introduce a patient-held critical care discharge summary [29] but these are not being used at present. This could be pursued in future PDSA cycles. While the patient story-telling work was not used to assess change, anecdotal reports from staff suggest that these are a powerful motivator and it is recommended that the utility of this as a behaviour change intervention is assessed via primary / implementation science research. This quality improvement project could have benefited from involvement of patients in the project steering group. This would help to improve the likelihood that the project benefits patients and is sustainable.

The ten month collaboration between researchers at KIS and clinical staff at $\mathrm{KCH}$ worked well. Regular contact between KIS and iMobile was essential. Monthly meetings with the whole project team were useful to update on progress and create actions with further meetings and email / phone contact as necessary. Organisational support for the project was helpful. The strengths of KIS lay in the 'planning' and 'studying' elements of the PDSA cycle; particularly: project management and administrative support, literature reviewing to ensure that the project was evidence based, construction of the process maps, collection of patient stories, descriptive analysis of the data, and drafting the quality improvement report. The iMobile team were invaluable in the 'doing' 
and 'acting' phases of the PDSA cycles; specifically: providing indepth knowledge about the service and local context; using clinical judgement in the conduct of audits, delivering educational sessions and local championing, and facilitating KIS team members' access to patients and staff. While contact between KIS and iMobile was regular, the collaboration may have been further strengthened through an even closer commitment to the 'researcher in residence' model whereby a researcher becomes embedded within the team.[30]

In the future, we are aware of the need for strong championing of this quality improvement project to prevent it from halting in favour of other endeavours or succumbing to the vast variety of local pressures. Unlike discharge summaries that are written at the point of discharge back into the community, there are no formal targets for the completion of critical care discharge summaries aside from the targets set by this project which may make it difficult to drive sustainability. The KIS team therefore propose to re-visit this project (and re-audit in full) in six months' time in order to maintain momentum.

Overall, the Model for Improvement [31] was a helpful approach to planning the processes involved in this quality improvement project. We supplemented PDSA cycles with project management techniques and additional quality improvement tools such as process mapping and patient story-telling.

Finally, we found the SQUIRE guidelines for reporting quality improvement projects to be helpful for both designing and reporting the results of this quality improvement project and we recommend their use by other teams.[32]

\section{Conclusion}

The audit results were mixed across the trajectory of the project, demonstrating the difficulty of sustaining positive change. Discharge is part of a complex system, thus a number of inter-related contextual factors are likely to underpin the quality of discharge from critical care, including staff rotation and the level of operational strain. We recommend that future PDSA cycles and quality improvement projects adopt a behaviour change theory or framework that introduces interventions designed to address the various barriers to improvement; and that continuous measurement is employed.

\section{References}

1. NICE clinical guideline 83 'Rehabilitation after critical illness' (2009). Available from: http://www.nice.org.uk/guidance/cg83. Accessed 02/02/15.

2. McKinney $A A$, and Deeny $P$. Leaving the intensive care unit: a phenomenological study of the patients' experience. Crit Care Nurs 2002;18:320-31.

3. Leith BA. Transfer anxiety in critical care patients and their family members. Crit Care Nurs 1999;18:24-32.

4. Chaboyer W, Kendall E, Kendall M, Foster M. Transfer out of Intensive care: an exploration of patient and family perceptions. Aust J Crit Care 2005;18(4):138-45.

5. Field K, Prinjha S \& Rowan K. One patient amongst many: a qualitative analysis of intensive care unit patients' experiences of transferring to the general ward. Crit Care 2008;12 (1): doi:10.1186/cc6795.

6. James S, Quirke S, McBride-Henry K. Staff perception of patient discharge from ICU to ward-based care. Nurs Crit Care 2013;18(6):297-306. doi: 10.1111/nicc.12001. Epub 2013 Jan 20.

7. Whittaker J, Ball C. Discharge from intensive care: a view from the ward. Crit Care Nurs 2000;16:135-43.

8. Australian Commission on Quality and Safety in Healthcare (2005) Clinical handover and patient safety: a literature review report. Available from: http://www.safetyandquality.gov.au/wpcontent/uploads/2012/01/clinhovrlitrev.pdf. Last accessed 02/02/15

9. Stelfox HT, Perrier L, Straus et al. Identifying intensive care unit discharge planning tools: protocol for a scoping review. BMJ Open 2013; doi:10.1136/bmjopen-2013-002653.

10. Petersen LA, Brennan TA, O'Neil AC, et al. Does housestaff discontinuity of care increase the risk for preventable adverse events? Ann Intern Med 1994;121:866-72.

11. Arora V, Johnson J, Lovinger D, et al. Communication failures in patient sign-out and suggestions for improvement: a critical incident analysis. Qual Saf Health Care 2005;14:401-7.

12. Shojania KG, Fletcher KE, Saint S. Graduate medical education and patient safety: a busy-and occasionally hazardous-intersection. Ann Intern Med 2006;145:592-8.

13. Apker J, Mallak LA, Gibson SC. Communicating in the "gray zone": perceptions about emergency physician hospitalist handoffs and patient safety. Acad Emerg Med 2007;14:884-94.

14. Stelfox HT, Lane D, Boyd JM (2014) A Scoping Review of Patient Discharge from Intensive Care: Opportunities and Tools to Improve Care. Chest. Online first, available from: http://journal.publications.chestnet.org/data/Journals/CHES T/932713/chest 147_2 317.pdf. Accessed 02/02/15.

15. NICE clinical guideline 50 'Acutely ill patients in hospital' (2007). Available from: http://www.nice.org.uk/guidance/cg50/resources/guidanceacutely-ill-patients-in-hospital-pdf. Accessed 02/02/15.

16. Australian Commission on Safety and Quality in Healthcare (2011) Electronic Discharge Summary Systems SelfEvaluation Toolkit. Available from: http://www.safetyandquality.gov.au/wpcontent/uploads/2012/01/EDS-self-eval-toolkit-sept2011.pdf. Accessed 02/02/15.

17. Rx for Change database (2014). Available from: https://www.cadth.ca/resources/rx-forchange/database/browse. Accessed 02/02/15.

18. Jamtvedt G, Young JM, Kristoffersen DT, O'Brien MA, Oxman AD. Audit and feedback: effects on professional practice and health care outcomes. Cochrane Database Syst Rev 2006; 2:CD000259.

19. Davey P, Brown E, Fenelon L, Finch R, Gould I, Hartman 
G, Holmes A, Ramsay C, Taylor E, Wilcox M, Wiffen P. Interventions to improve antibiotic prescribing practices for hospital inpatients. Cochrane Database Syst Rev 2005; 4:CD003543.

20. Dwamena F, Holmes-Rovner M, Gaulden CM, Jorgenson S, Sadigh G, Sikorskii A, Lewin S, Smith RC, Coffey J, Olomu A. Interventions for providers to promote a patientcentred approach in clinical consultations. Cochrane Database Syst Rev 2012; 12:CD003267.

21. Moore PM, Rivera MS, Grez AM, Lawrie TA. Communication skills training for healthcare professionals working with people who have cancer. Cochrane Database Syst Rev 2013; 3:CD003751.

22. Flodgren G, Parmelli E, Doumit G, Gattellari M, O'Brien MA, Grimshaw J, Eccles MP. Local opinion leaders: effects on professional practice and health care outcomes. Cochrane Database Syst Rev 2011; 8:CD000125.

23. Thomas L, Cullum N, McColl E, Rousseau N, Soutter J, Steen N. Guidelines in professions allied to medicine. Cochrane Database Syst Rev 2000; 2:CD000349.

24. Nkansah N, Mostovetsky O, Yu C, Chheng T, Beney J, Bond $\mathrm{C}$, Bero L. Effect of outpatient pharmacists' nondispensing roles on patient outcomes and prescribing patterns. Cochrane Database Syst Rev 2010; 7:CD000336.

25. O'Brien MA, Rogers S, Jamtvedt G, Oxman AD, OdgaardJensen J, Kristoffersen DT, Forsetlund L, Bainbridge D, Freemantle N, Davis DA, Haynes RB, Harvey EL. Educational outreach visits: effects on professional practice and health care outcomes. Cochrane Database Syst Rev 2007; 4:CD00040.

26. Arnold SR, Straus SE. Interventions to improve antibiotic prescribing practices in ambulatory care. Cochrane Database Syst Rev 2005; 4:CD003539.

27. Forsetlund L, Bjorndal A, Rashidian A, Jamtvedt G, O'Brien MA, Wolf F, Davis D, Odgaard-Jensen J, Oxman AD. Continuing education meetings and workshops: effects on professional practice and health care outcomes. Cochrane Database Syst Rev 2009; 2:CD003030.

28. Grimshaw JM, Shirran L, Thomas R, Mowatt G, Fraser C, Bero L, Grilli R, Harvey E, Oxman A, O'Brien MA. Changing provider behavior: an overview of systematic reviews of interventions. Med Care 2001; Aug;39 (8 Suppl 2):II2-45.

29. Bench S, Day D, Tina L, Griffiths P (2012) User centred critical care discharge information to support early critical illness rehabilitation; development and evaluation using the Medical Research Council's complex interventions framework. Available from: http://eprints.soton.ac.uk/300532/ Accessed: 02/02/15.

30. Marshall M, Pagel C, French C, Utley M, Allwood D, Fulop N, Pope C, Banks V, Goldmann A. Moving improvement research closer to practice: The researcher in residence model. BMJ Qual Saf 2014; 23: 801-5.

31. Institute for Healthcare Improvement (2014) Model for Improvement. Available from:

http://www.ihi.org/resources/Pages/Howtolmprove/default.a spx. Accessed 02/02/15.

32. Ogrinc G, Mooney SE, Estrada C, et al. The SQUIRE (Standards for QUality Improvement Reporting Excellence) guidelines for quality improvement reporting: explanation and elaboration. BMJ Qual Saf 2008;17(Suppl I):i13-i32. doi:10.1136/qshc.2008.029058.

\section{Declaration of interests}

Nothing to declare.

\section{Acknowledgements}

Grateful thanks are due to the staff and patients at King's College Hospital NHS Foundation Trust. Nurses from the iMobile team completed the data collection for the audits. Graham Thornicroft and Tracey Power from King's Improvement Science and Julia Wendon from King's College Hospital provided advice to the project team.

King's Improvement Science (KIS) comprises a specialist team of improvement scientists and senior researchers at King's College London. They help health professionals and managers who work in NHS services in south-east London to carry out quality improvement projects, and they also study the effectiveness of different improvement methods to ascertain which work best. KIS was set up in 2013 by King's Health Partners (an academic health science centre). Its work is funded by the four King's Health Partners organisations (Guy's and St Thomas' NHS Foundation Trust, King's College Hospital NHS Foundation Trust, King's College London and South London and Maudsley NHS Foundation Trust), Guy's and St Thomas' Charity, the Maudsley Charity and the Health Foundation. In 2014, KIS became part of the National Institute for Health Research (NIHR) Collaboration for Leadership in Applied Health Research and Care South London (CLAHRC South London): using CLAHRC funding, researchers at both King's College London and St George's, University of London, are working with NHS organisations, healthcare professionals, patients and service users to improve health services in south-west as well as south-east London.

The research was supported by the National Institute for Health Research (NIHR) Collaboration for Leadership in Applied Health Research and Care South London at King's College Hospital NHS Foundation Trust. The views expressed are those of the author(s) and not necessarily those of the NHS, the NIHR or the Department of Health.

\section{Ethical approval}

The project protocol was reviewed by the trust's research governance signatory for service evaluation studies and the project was deemed to be a service evaluation study. The project was given trust authorisation to proceed in accordance with the local governance requirements for service evaluation studies; therefore, the local policy meant that ethical approval was not required. 